Review Article

\title{
Prevention and Treatment of Osteoporosis Using Chinese Medicinal Plants: Special Emphasis on Mechanisms of Immune Modulation
}

\author{
Hongyan Zhao $\mathbb{D D}^{1,2}$ Ning Zhao, ${ }^{3}$ Peng Zheng, ${ }^{4}$ Xiaohong Xu, ${ }^{5}$ Meijie Liu, ${ }^{1,2}$ Dan Luo, ${ }^{6}$ \\ Huihui Xu $\mathbb{B}^{1},{ }^{1}$ and Dahong Ju (i) ${ }^{1,2}$ \\ ${ }^{1}$ Experimental Research Center, China Academy of Chinese Medical Sciences, Beijing 100700, China \\ ${ }^{2}$ Institute of Basic Theory for Chinese Medicine, China Academy of Chinese Medical Science, Beijing 100700, China \\ ${ }^{3}$ Institute of Clinical Basic Medicine, China Academy of Chinese Medical Sciences, Beijing 100700, China \\ ${ }^{4}$ Jilin Provincial Hospital of Traditional Chinese Medicine, Changchun University of Chinese Medicine, Changchun 130021, China \\ ${ }^{5}$ Changchun University of Chinese Medicine, Changchun 130117, China \\ ${ }^{6}$ Traditional Chinese Medicine Hospital of Changping District, Beijing 102200, China
}

Correspondence should be addressed to Dahong Ju; judahong@126.com

Received 24 August 2017; Revised 5 November 2017; Accepted 6 December 2017; Published 20 February 2018

Academic Editor: Wuxiang Xie

Copyright (C) 2018 Hongyan Zhao et al. This is an open access article distributed under the Creative Commons Attribution License, which permits unrestricted use, distribution, and reproduction in any medium, provided the original work is properly cited.

\begin{abstract}
Numerous studies have examined the pathogenesis of osteoporosis. The causes of osteoporosis include endocrine factors, nutritional status, genetic factors, physical factors, and immune factors. Recent osteoimmunology studies demonstrated that the immune system and immune factors play important regulatory roles in the occurrence of osteoporosis, and people should pay more attention to the relationship between immunity and osteoporosis. Immune and bone cells are located in the bone marrow and share numerous regulatory molecules, signaling molecules, and transcription factors. Abnormal activation of the immune system alters the balance between osteoblasts and osteoclasts, which results in an imbalance of bone remodeling and osteoporosis. The incidence of osteoporosis is also increasing with the aging of China's population, and traditional Chinese medicine has played a vital role in the prevention and treatment of osteoporosis for centuries. Chinese medicinal plants possess unique advantages in the regulation of the immune system and the relationships between osteoporosis and the immune system. In this review, we provide a general overview of Chinese medicinal plants in the prevention and treatment of osteoporosis, focusing on immunological aspects.
\end{abstract}

\section{Introduction}

Osteoporosis is a systemic skeletal disease characterized by a decrease in bone mass and microstructural degradation, which may increase bone fragility and fracture danger and consequently cause serious complications [1]. The etiology of osteoporosis is primarily concentrated in endocrine and metabolic disorders. However, osteoimmunology demonstrated that the immune system and immune factors may be involved in the development of osteoporosis and play important regulatory roles $[2,3]$. The microenvironment created by bone cells provides conditions for development of the immune system, which regulates bone metabolism via B cells,
T cells, dendritic cells, and many cytokines [2-5]. Abnormal activation of the immune system alters the balance between osteoblasts and osteoclasts, which results in an imbalance in bone remodeling and causes osteoporosis [6-8].

Osteoporosis seriously affects human health, and there is no safe and effective method to restore lost bone. However, Chinese medicinal plants reduce bone loss and treat osteoporosis $[9,10]$. Chinese medicinal plants possess unique advantages in the regulation of the immune system [11-18] and alter the relationships between osteoporosis and the immune system [3, 4, 7]. This review summarizes the progress of Chinese medicinal plants in the prevention and treatment of osteoporosis, focusing on the immunological aspects. 


\section{Immune System and Osteoporosis}

The immune system is an important regulator of bone turnover. Therefore, the relationship between the immune system and bone is generally defined as "osteoimmunology." Osteoimmunology is a new interdisciplinary research field that focuses on the interactions between the immune system and bone at the anatomical, vascular, cellular, and molecular levels [19-21]. Osteoimmunology provides new pathogenetic and clinical interpretations for osteoporosis [6]. Many signaling factors and cytokines are involved in this field [22]. However, osteoprotegerin (OPG)/the receptor activator of nuclear factor-kappa B (RANK)/RANK ligand (RANKL) signaling is a key vinculum that is essential for osteoclastogenesis and immune regulation [4, 23]. Proinflammatory cytokines, such as tumor necrosis factor(TNF-) $\alpha$, interleukin- (IL-) 1 , and IL-17, also play pivotal roles in osteoimmunology [24]. Therefore, abnormalities of the immune system may cause bone diseases, such as osteoporosis and inflammatory bone loss [25]. Balancing the immune response promotes the formation of bone tissue, so as to improve osteoporosis.

2.1. RANK/RANKL/OPG Signaling Pathway. A functional imbalance between osteoblasts and osteoclasts causes osteoporosis. As a hallmark of osteoporosis, the increased activation of osteoclasts results in the progressive loss of bone mass and an increased susceptibility to bone fractures. RANKL is a TNF family cytokine that is primarily expressed by osteoblasts and also secreted by bone marrow stromal cells (BMSCs), preosteoblasts, osteocytes, fibroblasts, and cells of the immune system, including antigen-stimulated $\mathrm{T}$ cells and mature dendritic cells. Osteoclast differentiation is initiated via the binding of RANKL to RANK [26]. RANKLRANK interaction activates the master transcription factor for osteoclastogenesis; nuclear factor of activated $\mathrm{T}$ cells, cytoplasmic 1 (NFATc1); tartrate-resistant acid phosphatase; calcitonin receptor; and cathepsin $\mathrm{K}$ [27]. OPG is produced by osteoblasts, BMSCs, B cells, and dendritic cells, is a soluble decoy receptor for RANKL, and prevents RANKL from binding to RANK on the surface of osteoclastic lineage cells $[28,29]$. The relative ratio of RANKL to OPG is the critical determinant and the final step in the regulation of osteoclast biology and bone resorption. Therefore, dysregulation of the immune system may affect bone remodeling, and the immune system regulates bone metabolism primarily via the RANK/RANKL/OPG signaling pathway [30-33]. Various osteotropic hormones, cytokines, and drugs modulate the RANKL-to-OPG ratio [32, 34, 35].

\subsection{Immune Cell}

2.2.1. T Cells. The primary pathological process of osteoporosis is the imbalance of bone resorption and bone formation during bone remodeling. $\mathrm{T}$ cells regulate bone cells and hematopoiesis in the immune system, and these cells secrete inflammatory factors and Wnt ligands to promote bone formation and absorption $[2,3,36]$. T cells also regulate the dynamic balance of metabolism between bone mesenchymal cells and osteoblasts via CD40 ligands and costimulatory molecules [37]. However, the stimulating and inhibiting effects of $\mathrm{T}$ cells are related to their subsets, cytokines, and local factors.

$\mathrm{T}$ cells may be generally classified as effector-cytotoxic $\mathrm{T}$ cells $\left(\mathrm{CD}^{+}\right.$cells) and Thelper (Th) cells $\left(\mathrm{CD} 4^{+}\right.$cells). $\mathrm{CD} 4^{+}$ $\mathrm{T}$ cells develop into diverse Th cell subsets on activation and expansion and secrete signature cytokine profiles and mediate distinct effector functions [38]. T cells were divided into Th1 or Th2 cells depending on the cytokines produced, with Th1 producing IFN- $\gamma$ and IL- 2 and Th2 producing primarily IL-4/IL-5/IL-10, until recently. Regulatory T cells (Tregs, $\mathrm{CD} 4^{+} \mathrm{CD} 25^{+} \mathrm{Foxp}^{+}$) exhibit immune suppressive effects in the immune system, regulate bone remodeling, and are closely linked to skeletal-related disease, such as osteoporosis and rheumatoid arthritis (RA) [39, 40]. Tregs suppress osteoclastogenesis via the production of transforming growth factor- (TGF-) $\beta$, IL-10, and IL-4 [41, 42] and also regulate osteoclast differentiation via the cytotoxic $\mathrm{T}$ lymphocyte antigen (CTLA-4) [43, 44].

A third subset of IL-17-producing effector Th cells, called Th17 cells, was more recently discovered and characterized. Th17 cells play an important role in the pathogenesis of osteoporosis and directly produce high levels of IL-17, RANKL, and TNF to promote the formation and activation of osteoclasts $[38,45,46]$. Th17 cell populations and IL-17 levels were obviously increased in an osteoporosis model of ovariectomized (OVX) animals. Transcription factors related to Th17 cell differentiation are highly expressed [47]. IL-17 significantly promotes the production of RANKL, IL-6, and TNF, which promote bone resorption via upregulation of osteoclast formation [48, 49]. Th17 cells are responsible for regulating osteoclastogenesis, especially in autoimmune arthritis.

2.2.2. B Cells. The role of $\mathrm{B}$ cells in osteoimmunological interactions has been a matter of great concern. To our knowledge, $B$ cells are active regulators of the RANK/RANKL/ OPG system [23]. Bone-forming osteoblasts and BMSCs have historically been considered an important source of OPG under conditions of physiological bone remodeling. However, some studies demonstrated that B cells and plasma cells in the bone microenvironment are significant sources of OPG [50-52], which is a neutralizing soluble decoy receptor that competes with RANKL, thus blocking the binding of RANKL and RANK, which eliminates the effect of RANKL on osteoclasts [53]. However, other studies also demonstrated that activated B cells contributed to joint destruction via RANKL expression [54-57]. Xu et al. further demonstrated that mechanistic target of rapamycin complex 1 activation-stimulated RANKL expression in B cells sufficiently induced bone loss and osteoporosis [58].

2.3. Cytokines. A variety of cytokines are involved in the pathogenic process of osteoporosis. Recent studies showed that women with low bone mineral density (BMD) exhibited a proresorptive cytokine bias [59]. The proresorptive cytokines TNF, IL-6, IL-12, and IL-17 were higher in women with low BMD compared to women with normal BMD, and the antiresorptive cytokines IL-4, IL-10, and IL-23 were lower 
in peripheral blood mononuclear cells (PBMCs). Estrogen decrease after menopause exerts the same stimulating effect, which increases osteoclastic activity via IL-1, IL-6, and TNF- $\alpha[60,61]$. IL-1 is a very important cytokine in osteoimmunological processes, and it acts as the primary stimulus of osteoclast-activating factor. TNF- $\alpha$ and IL- 6 possess the same bone resorbing stimulating activity, and these three cytokines increase the osteoclast response to RANKL, which leads to osteolysis. OPG/RANK/RANKL signaling is the most important vinculum for osteoclastogenesis, and most cytokines exert effects via this pathway. RANKL and macrophage colony-stimulating factor (M-CSF) are the minimal necessary cytokines required for osteoclast formation [61]. $\mathrm{M}-\mathrm{CSF}$ induces the proliferation and differentiation of osteoclast precursors and improves the survival of mature osteoclasts. Other inflammatory cytokines, such as IL-4, IL-10, IL-12, IL-13, IL-18, and interferon- $\gamma$, strongly inhibit osteoclastogenesis and reduce bone loss [62-65].

2.4. Autoimmune Diseases. Autoimmune diseases, such as RA, ankylosing spondylitis (AS), systemic lupus erythematous, and acquired immune deficiency syndrome, involve the damage to joints and bone. RA is characterized by synovial inflammation that results in severe juxta-articular bone erosions and systemic osteoporosis. Previous research showed a greater risk of osteoporotic fractures in RA patients across all age groups, sex, and various anatomic sites compared to non-RA patients [66]. Lower BMD of the lumbar spine and hip was reported in AS patients compared to a control group [67], and $21 \%$ of AS patients exhibited osteoporotic vertebral compression fractures, which was 5 times greater than the control population [68].

Associations between osteoclast and proinflammatory cytokines may partially explain the cause of osteoporosis in autoimmune diseases. Active immune cells at sites of inflammation produce proinflammatory and osteoclastogenic cytokines, which lead to bone erosions and periinflammatory and systemic bone loss. Proinflammatory cytokines, such as TNF- $\alpha$, IL-1, IL- 6 , and IL-17, are higher in RA patients, which increase RANKL expression and cause bone loss [69]. Peri-inflammatory bone formation is impaired, which results in nonhealing of erosions and a local vicious circle of inflammation between synovitis, osteitis, and local bone loss.

\section{Immunoregulation Mechanisms of Chinese Medicinal Plants in the Prevention and Treatment of Osteoporosis}

Many plants in China are used for the prevention and treatment of bone and joint diseases, such as bone pain, osteoporosis, osteoarthritis, and RA. Compounds consisting primarily of these plants, such as Liuwei Dihuang pills (LWDHP) and Zuo-Gui-Wan (ZGW), play important roles in clinical treatment. Many studies investigated the important role in prevention and treatment of osteoporosis [70], and rapid progress has been made in delineating the mechanisms. Therefore, we discuss and summarize the mechanism of some representative Chinese medicinal plants and compounds, especially immunoregulation (Table 1).

\subsection{Chinese Medical Plants and Its Monomer}

3.1.1. Sinomenium acutum (Thunb.) Rehd. et Wils. and SIN. Sinomenium acutum (Thunb.) Rehd. et Wils. has been used as a natural drug to treat rheumatic diseases in China for centuries. SIN was isolated from the root and stem of Sinomenium acutum (Thunb.) Rehd. et Wils. in the 1920s [71], and it exhibited good anti-inflammatory and immunoregulatory properties [72].

Liu et al. [73] found that SIN significantly inhibited $\left[{ }^{3} \mathrm{H}\right]-$ thymidine incorporation into mouse spleen cells activated with concanavalin A. The Th1-specific transcription factor T-bet is selectively expressed in Th1 cells and plays an important role in the development of Th1 cells via initiation of Th1 genetic processes and inhibition of the synthesis of Th2 cytokines. GATA-3 is a Th2 cell-specific transcription factor that is directly involved in the regulation of the differentiation of T lymphocytes, induces the generation of Th2 cells, eosinophil differentiation, and the regulation of eosinophil and natural killer cells; GATA-3 also inhibits the $\mathrm{CD} 8^{+} \mathrm{T}$ lymph cell differentiation and maturation. One clinical trial demonstrated that SIN decreased the expression of T-bet mRNA and IFN- $\gamma$ and regulated the ratio of T-bet and GATA-3 and Th1/Th2 cytokine balance in mesangial proliferative nephritis [74]. The novel derivatives of SIN directly inhibited Th17 cell differentiation and alleviated the inflammatory symptoms of experimental autoimmune encephalomyelitis [75].

SIN is widely used in the treatment of inflammatory diseases, especially RA. SIN significantly improved arthritis in rats via inhibition of synovial fibroblast proliferation and anti-type II collagen antibody levels $[76,77]$ and regulation of Th1/Th2 and the ratio of MMPs and their endogenous inhibitors, called TIMPs [77, 78]. Recent studies demonstrated that SIN modulated bone metabolism via suppression of osteoclastogenesis and related transduction signals. Li et al. demonstrated that SIN suppressed osteoclast formation and bone loss via modulation of RANKL signaling pathways [79] and Zhou et al. [80] revealed that SIN was a proinflammatory mediator that regulated the immunosuppressive effects of marrow stromal cells and inhibited osteoclast differentiation via inhibition of the $\mathrm{PGE}_{2}$-induced OPG/ RANKL ratio. Further studies revealed that SIN inhibited the activation and relative gene expression of NF- $\kappa \mathrm{B}, \mathrm{AP}-1$, and NFAT and downregulated phosphorylation of MAPK p38 in osteoclastogenesis via reduction of Toll-like receptor 4/TRAF6 expression [81]. Therefore, SIN may be a promising agent for the treatment of osteoporosis.

3.1.2. Davallia mariesii Moore ex Bak. and Naringin. Naringin is widely distributed in various types of plants, and it is a major component extracted from the Chinese medicinal herb Davallia mariesii Moore ex Bak. This herb exhibits inhibitory effects on inflammatory responses and bone destruction and anabolic effects on bone in clinical treatment. The experimental results confirmed that naringin inhibited 


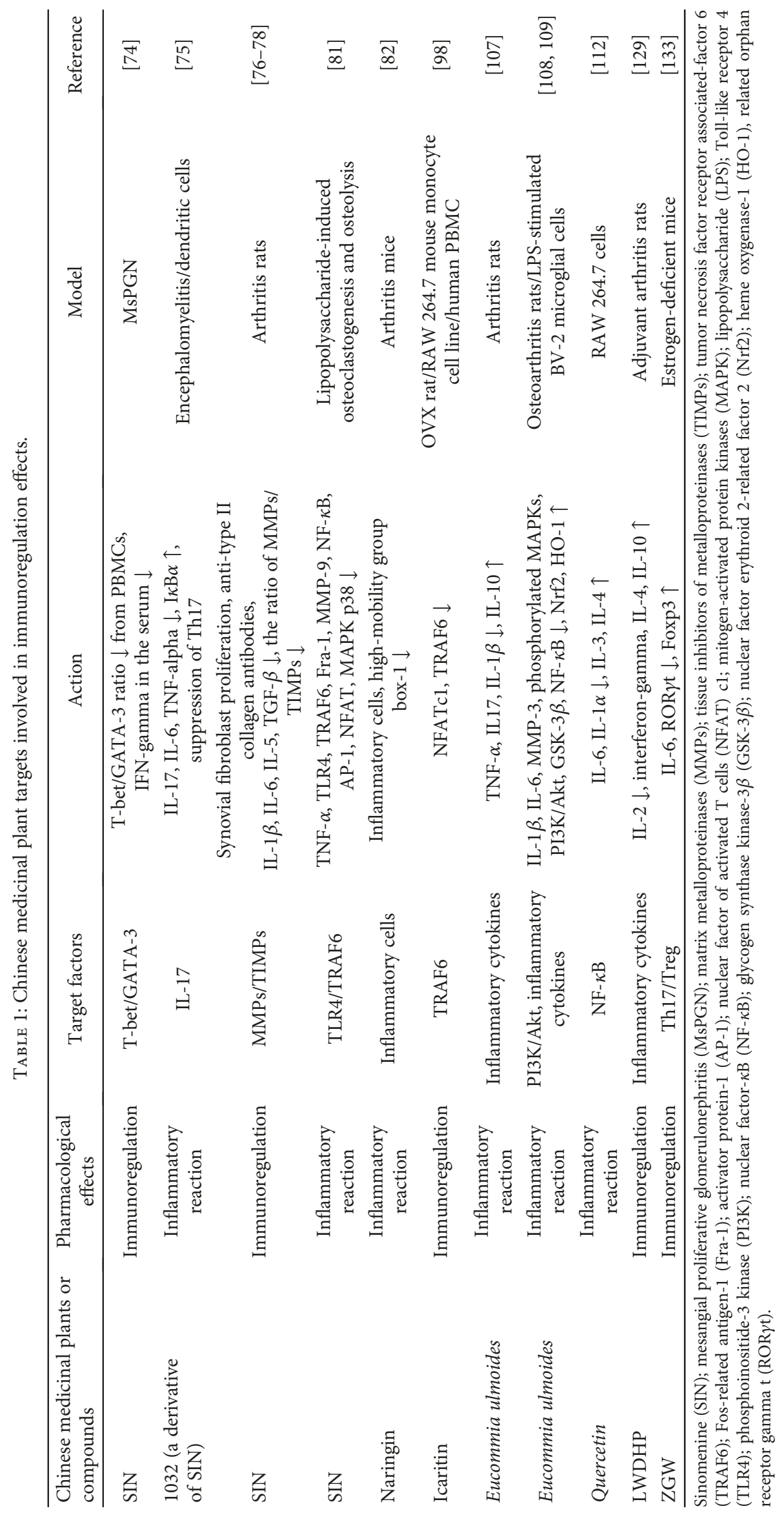


inflammation via reduction of inflammatory cytokine and NF- $\kappa$ B expression [82].

Naringin exhibited some therapeutic effects on osteoporosis in animal experiments. Naringin inhibited tartrateresistant acid phosphatase activity, decreased RANKL expression, inhibited osteoclast activity, reduced bone loss, and decreased BMD, which altered bone resorption. Further study demonstrated that naringin inhibited osteoclast activity via inhibition of RANK-mediated NF-kappaB and extracellular regulated protein kinase signaling [83]. Naringin increased osteoblast proliferation by increasing BMP-2 expression via PI3K, Akt, c-Fos/c-Jun, and the AP-1 pathway [84]. Wong et al. and Pang et al. also found that naringin increased osteopontin and osteoprotegerin expression and osteocalcin $[85,86]$. Naringin promoted the osteogenic differentiation of BMSCs by upregulating Foxc2 expression via the IHH signaling pathway [87].

3.1.3. Epimedium davidii Franch., Icariin, and Icaritin. Epimedium davidii Franch. has been used for centuries to treat osteoporosis, based on the function of "strengthening" bones. Icariin is the primary active flavonoid glucoside isolated from Epimedium davidii Franch., and it is highly related to the therapeutic effects of this herb. However, its bone-strengthening activity attracted much more attention in recent years. Icariin enhances antiosteoporotic activity by initiating osteoblastic differentiation and mineralization, inhibiting adipogenesis, preventing osteoclast differentiation, and inducing apoptosis of osteoclasts, which decrease bone resorption and bone loss [88-92]. Liu et al.'s research indicated that icariin suppressed the differentiation of mesenchymal stem cells into adipocytes via inhibition of peroxisome proliferator-activated receptor gamma, CCAAT/enhancer binding protein $\alpha$, fatty acidbinding protein $4 \mathrm{mRNA}$, N1ICD, and jagged1 protein expression and increasing Notch2 mRNA in OVX rats, which improved osteoporosis [93]. Further research indicated that icariin also promoted bone fracture healing in OVX osteoporotic rats [94, 95]. Icariin could increase the proliferation and matrix mineralization of osteoblasts and promote NO synthesis. Further study showed that with icariin treatment, the BMP-2, SMAD4, Cbfa1/Runx2, and OPG gene expressions were upregulated; the RANKL gene expression was however downregulated. This effect may contribute to its action on the induction of osteoblast proliferation and differentiation, resulting in bone formation [96]. Liang's results also indicated that icariin could promote bone formation via the BMP-2/Smad4 signal pathway in hFOB 1.19 cells then promote bone formation [97].

Icaritin, an internal metabolite of icariin, possesses similar function with icariin. Icaritin inhibited osteoclast formation via the downregulation of TRAF6 and coordinated inhibition of NF- $\kappa$ B, MAPK/AP-1, and reactive oxygen species signaling pathways to reduce NFATcl expression and activity in vitro and in an OVX rat [98]. Wu et al. and Sheng et al. indicated that icaritin enhanced the osteogenic differentiation of derived mesenchymal stem cells and human adipose tissue-derived stem cells by increasing the protein levels of BMPs, Runx2, and osteocalcin [99] and inhibiting adipogenesis of marrow mesenchymal stem cells via suppression of glycogen synthase kinase- $3 \beta$ and peroxisome proliferator-activated receptor gamma [100].

3.1.4. Eucommia ulmoides and Quercetin. Eucommia ulmoides is a kidney-tonifying herbal medicine in China with a long history for treatment of bone and joint diseases, such as osteoporosis, osteoarthritis, and RA. Eucommia ulmoides and its extracts participate in bone metabolism via activation of osteoblasts to facilitate osteogenesis and suppression of osteoclast activity to inhibit osteolysis [101]. In vivo studies showed that extracts of Eucommia ulmoides improve bone biomechanical quality via modification of BMD and trabecular microarchitecture in OVX rats [102-105]. In vitro evidence suggests that extracts of Eucommia ulmoides could induce primary osteoblastic cell proliferation and differentiation and inhibit osteoclastogenesis via an increase in OPG and decrease in RANKL expression [106].

An ethanol extract of Eucommia ulmoides relieved arthritis degradation via inhibition of the key proinflammatory cytokines TNF- $\alpha$, IL17, and IL- $1 \beta$ and increases the antiinflammatory effects of IL-10 [107]. The PI3K/Akt pathway may play an important role in this process. Eucommia ulmoides also inhibited the progression of osteoarthritis via inhibition of the PI3K/Akt pathway to reduce inflammatory cytokines, bone destruction, and bone loss [108, 109].

The flavonol glycoside quercetin was isolated from the leaves of Eucommia ulmoides, and its structure was identified using NMR and ESIMS analyses [110]. Quercetin exhibited soluble epoxide hydrolase inhibitory activity and antiinflammatory properties, partially because of its capacity to downmodulate the NF- $\kappa$ B signal transduction pathway [111-114]. Quercetin suppressed osteoclastogenesis in vitro and prevented bone loss in OVX mice in vivo $[115,116]$. Yamaguchi and Weitzmann found that quercetin potently suppressed osteoclastogenesis and RANKL-induced NF- $\kappa \mathrm{B}$ activation in osteoclast precursors [117]. Guo et al. also found that quercetin inhibited RANK, TRAF6, and COX-2 expression, induced apoptosis, and inhibited bone resorptive activity in LPS-induced mature osteoclasts. Quercetin promoted the apoptotic signaling pathway, including increasing the phosphorylation of p38-MAPK, c-Jun N-terminal kinases/ stress-activated protein kinases (JNK/SAPK), and Bax and inhibited Bcl-2 expression [118]. Some studies demonstrated that quercetin exerted a stimulatory effect on bone formation and reversed the inhibition of osteoblast differentiation induced by LPS via MAPK signaling in vitro $[119,120]$.

3.1.5. Other Chinese Medicinal Plants and Monomers. Many Chinese medicinal plants and monomers effectively treat osteoporosis. Echinacoside is isolated from Cistanchetubulosa (Schrenk) R. Wight stems, and it exhibited notable antiosteoporotic effects in an OVX rat model of osteoporosis and enhanced bone regeneration in MC3T3-E1 cells in vitro [121]. Radix Dipsaci has long been used as an antiosteoporotic drug. Radix Dipsaci total saponins are the main active ingredient of Radix Dipsaci, and this component effectively suppressed the loss of bone mass in OVX rats [122]. Achyranthes bidentata Blume. improved bone biomechanical 
quality via modifications of BMD6 and trabecular microarchitecture, which prevented OVX-induced osteoporosis in rats [123]. Achyranthes bidentata Blume. and Panax notoginseng saponin are the main active components of Panax notoginseng that prevent OVX-induced osteoporosis via enhancement of BMD, bone strength, and prevention of the deterioration of trabecular microarchitecture in OVX osteoporosis rats [124]. Diarylheptanoid from Curcuma comosa Roxb. decreased NFATc1 and c-Fos expression via the MAPK pathway and inhibited RANKL-induced osteoclast differentiation [125]. The natural compound Polygonatum sibiricum polysaccharide promoted osteoblastic differentiation and mineralization via the ERK/GSK-3 $\beta / \beta$-catenin signaling pathways [126]. These studies provide a therapeutic approach for the use of Chinese plants in the prevention of osteoporosis.

\subsection{Compound Recipes of Medicinal Plants}

3.2.1. LWDHP. As a classic formula of traditional Chinese medicine, LWDHP has been used to prevent and treat various diseases with characteristic features of kidney yin deficiency in China for more than 1000 years. LWDHP is widely prescribed as therapy or adjuvant therapy for various diseases, such as menopause, osteoporosis, and diabetes. LWDHP exhibits a wide range of pharmacological effects via regulation of the balance of the neuroendocrine immunomodulation network [127].

LWDHP exhibits bidirectional regulation to the immune system. LWDHP exerted its pharmacological action via immunomodulation rather than immunosuppression, which differs from Cy and CsA [128]. Jian et al. demonstrated that LWDHP significantly inhibited the mRNA expression of IL-1 (Th1 cytokines) and promoted the expression of IFN- $\gamma$ (Th1 cytokines), IL-4 (Th2 cytokines), and IL-10 (Th2 cytokines) in splenocytes of AA rats. These results indicated that LWDHP enhanced the function of splenic Th cells via restoration of Th1 and Th2 cytokine levels and modulation of the balance of the Th1/Th2 [129]. LWDHP also significantly improved the function of $\mathrm{T}$ and $\mathrm{B}$ lymphocytes in aging mice and corrected the imbalance of $\mathrm{CD}^{+} /$ $\mathrm{CD}^{+} \mathrm{T}$ cells in the spleen.

The mechanism of LWDHP treatment of osteoporosis is also related to its immunomodulatory function. The JAK/ STAT signaling pathway plays a vital role in bone metabolism. LWDHP regulates immune function by increasing the expression of the immune-related gene cardiotrophin-like cytokine factor 1 and activation of the JAK/STAT signaling pathway to treat postmenopausal osteoporosis with kidney yin deficiency [130]. Xia et al. suggested that LWDHP could alleviate osteoporosis partially via a significant enhancement in the levels of Lrp-5, $\beta$-catenin, Runx2, and Osx, which were involved in the canonical $\mathrm{Wnt} / \beta$-catenin signaling pathway of osteoblasts, in osteoporosis model induced by ovariectomy and in vitro experiments [131].

3.2.2. $Z G W$. The $Z G W$ is another classical traditional Chinese medicine (TCM) herbal prescription for tonifying kidney yin. This prescription was recorded in Jing yue Quan shu, which is a famous TCM book, in 1624 A.D.

Our results showed that the ZGW significantly increased the expression levels of Wnt1, low-density lipoprotein receptor-related protein 5 , and beta-catenin proteins in osteoblasts and BMSCs of glucocorticoid-induced osteoporosis rats after eight weeks of administration [132]. These results indicated that the ZGW prevented and treated osteoporosis, and the Wnt signal transduction pathway played an important role in this progress. The Th17/Treg paradigm plays a vital role in the regulation of bone metabolism. Lai et al. found that the ratio of Th17 and Treg shifted to Th17 in estrogen-deficient OVX and aged mice. Treatment with ZGW markedly enhanced BMD, decreased IL- 6 and ROR $\gamma \mathrm{t}$ expression, and significantly increased the level of Foxp3. These results indicated that the ZGW could prevent bone loss, and the mechanism was a Th17/Treg paradigm that skewed towards Treg [133].

\section{Conclusion}

Many studies investigate the pathogenesis of osteoporosis, and the immunological regulation of osteoporosis is a new research hotspot that provides a new mechanism of the pathogenesis of osteoporosis. Immune and bone cells exist together in the microenvironment of bone cavities, and these cells share a variety of regulatory molecules. Many cytokines in bone metabolism play a very important role in the proliferation, differentiation, and activation of osteoclasts and osteoblasts. However, the specific mechanisms of these cytokines have not reached an indisputable conclusion, and the role of many cytokines in the process of bone metabolism is not clear.

The clinical efficacy of Chinese medicinal plants has shown their advantages in the treatment of osteoporosis. An increasing number of studies demonstrated that these plants play an important role in the treatment of osteoporosis by regulating the immune system, but more research is required. Notably, the mechanism of action of traditional Chinese medicine is not a single pathway, but it is multiroute and multitargeted. Therefore, its regulation of the immune system cannot be the only mechanism for the treatment of osteoporosis. Our continued improvement in understanding of the immunoregulatory mechanisms of osteoporosis will further elucidate the immunoregulatory mechanism of Chinese medicine for the treatment of osteoporosis.

\section{Conflicts of Interest}

The authors declare no conflicts of interest.

\section{Authors' Contributions}

Hongyan Zhao, Ning Zhao, and Dahong Ju participated in the design. Hongyan Zhao, Ning Zhao, Peng Zheng, Xiaohong Xu, Meijie Liu, Dan Luo, and Huihui Xu wrote the manuscript. Dahong Ju edited the manuscript. All of the authors approved the manuscript. Hongyan Zhao and Ning Zhao contributed equally to this work. 


\section{Funding}

This work was supported by the National Natural Science Foundation of China (Grant nos. 81373773, 81573845, 81673841, and 81673844).

\section{References}

[1] J. P. van den Bergh, T. A. van Geel, and P. P. Geusens, "Osteoporosis, frailty and fracture: implications for case finding and therapy," Nature Reviews Rheumatology, vol. 8, no. 3, pp. 163-172, 2012.

[2] A. Limmer and D. C. Wirtz, "Osteoimmunology: influence of the immune system on bone regeneration and consumption," Zeitschrift für Orthopädie und Unfallchirurgie, vol. 155, no. 03, pp. 273-280, 2017.

[3] M. N. Weitzmann and I. Ofotokun, "Physiological and pathophysiological bone turnover-role of the immune system," Nature Reviews Endocrinology, vol. 12, no. 9, pp. 518-532, 2016.

[4] A. Bozec and M. M. Zaiss, "T regulatory cells in bone remodelling," Current Osteoporosis Reports, vol. 15, no. 3, pp. 121-125, 2017.

[5] L. Zhao, L. Huang, and X. Zhang, "Osteoimmunology: memorandum for rheumatologists," Science China. Life Sciences, vol. 59, no. 12, pp. 1241-1258, 2016.

[6] H. Takayanagi, "Osteoimmunology and the effects of the immune system on bone," Nature Reviews Rheumatology, vol. 5, no. 12, pp. 667-676, 2009.

[7] M. M. Guerrini and H. Takayanagi, "The immune system, bone and RANKL," Archives of Biochemistry and Biophysics, vol. 561, pp. 118-123, 2014.

[8] T. Nakashima and H. Takayanagi, "Osteoimmunology: crosstalk between the immune and bone systems," Journal of Clinical Immunology, vol. 29, no. 5, pp. 555-567, 2009.

[9] Z. Q. Wang, J. L. Li, Y. L. Sun et al., "Chinese herbal medicine for osteoporosis: a systematic review of randomized controlled trails," Evidence-based Complementary and Alternative Medicine, vol. 2013, Article ID 356260, 11 pages, 2013.

[10] J. B. He, M. H. Chen, and D. K. Lin, "New insights into the tonifying $\mathrm{k}$ ' in herbs and formulas for the treatment of osteoporosis," Archives of Osteoporosis, vol. 12, no. 1, p. 14, 2017.

[11] L. Tong and K. D. Moudgil, "Celastrus aculeatus Merr. suppresses the induction and progression of autoimmune arthritis by modulating immune response to heat-shock protein 65," Arthritis Research \& Therapy, vol. 9, no. 4, article R70, 2007.

[12] A. Ma, Y. Yang, Q. Wang, Y. Wang, J. Wen, and Y. Zhang, "Antiinflammatory effects of oxymatrine on rheumatoid arthritis in rats via regulating the imbalance between Treg and Th17 cells," Molecular Medicine Reports, vol. 15, no. 6, pp. 3615-3622, 2017.

[13] T. Chen, S. Yuan, X. N. Wan et al., "Chinese herb cinobufagin-reduced cancer pain is associated with increased peripheral opioids by invaded $\mathrm{CD}^{3 / 4 / 8}$ lymphocytes," Oncotarget, vol. 8, no. 7, pp. 11425-11441, 2017.

[14] S. Y. Park, S. J. Jung, K. C. Ha et al., “Anti-inflammatory effects of Cordyceps mycelium (Paecilomyces hepiali, CBG-CS-2) in Raw264.7 murine macrophages," Oriental Pharmacy and Experimental Medicine, vol. 15, no. 1, pp. 7-12, 2015.
[15] C. Xiao, L. Zhao, Z. Liu et al., "The effect of triptolide on CD4+ and CD8+ cells in the Peyer's patch of DA rats with collagen induced arthritis," Natural Product Research, vol. 23, no. 18, pp. 1699-1706, 2009.

[16] L. J. Ho and J. H. Lai, "Chinese herbs as immunomodulators and potential disease-modifying antirheumatic drugs in autoimmune disorders," Current Drug Metabolism, vol. 5, no. 2, pp. 181-192, 2004.

[17] K. Asano, J. Matsuishi, Y. Yu, T. Kasahara, and T. Hisamitsu, "Suppressive effects of Tripterygium wilfordii Hook $f$., a traditional Chinese medicine, on collagen arthritis in mice," Immunopharmacology, vol. 39, no. 2, pp. 117-126, 1998.

[18] A. F. Tawfik, S. J. Bishop, A. Ayalp, and F. S. el-Feraly, "Effects of artemisinin, dihydroartemisinin and arteether on immune responses of normal mice," International Journal of Immunopharmacology, vol. 12, no. 4, pp. 385-389, 1990.

[19] H. Takayanagi, "Osteoimmunology: shared mechanisms and crosstalk between the immune and bone systems," Nature Reviews Immunology, vol. 7, no. 4, pp. 292-304, 2007.

[20] M. B. Greenblatt and J. H. Shim, "Osteoimmunology: a brief introduction," Immune Network, vol. 13, no. 4, pp. 111-115, 2013.

[21] H. Takayanagi, "Osteoimmunology in 2014: two-faced immunology-from osteogenesis to bone resorption," Nature Reviews Rheumatology, vol. 11, no. 2, pp. 74-76, 2015.

[22] W. J. Boyle, W. S. Simonet, and D. L. Lacey, "Osteoclast differentiation and activation,” Nature, vol. 423, no. 6937, pp. 337-342, 2003.

[23] M. C. Walsh and Y. Choi, "Biology of the RANKL-RANKOPG system in immunity, bone, and beyond," Frontiers in Immunology, vol. 5, p. 511, 2014.

[24] L. Ginaldi and M. De Martinis, "Osteoimmunology and beyond," Current Medicinal Chemistry, vol. 23, no. 33, pp. 3754-3774, 2016.

[25] G. Mori, P. D'Amelio, R. Faccio, and G. Brunetti, "The interplay between the bone and the immune system," Clinical and Developmental Immunology, vol. 2013, Article ID 720504, 16 pages, 2013.

[26] T. Ikeda, M. Utsuyama, and K. Hirokawa, "Expression profiles of receptor activator of nuclear factor $\kappa \mathrm{B}$ ligand, receptor activator of nuclear factor $\kappa \mathrm{B}$, and osteoprotegerin messenger RNA in aged and ovariectomized rat bones," Journal of Bone and Mineral Research, vol. 16, no. 8, pp. 1416-1425, 2001.

[27] J. H. Kim and N. Kim, "Regulation of NFATc1 in osteoclast differentiation," Journal of Bone Metabolism, vol. 21, no. 4, pp. 233-241, 2014.

[28] D. L. Lacey, E. Timms, H. L. Tan et al., "Osteoprotegerin ligand is a cytokine that regulates osteoclast differentiation and activation," Cell, vol. 93, no. 2, pp. 165-176, 1998.

[29] W. S. Simonet, D. L. Lacey, C. R. Dunstan et al., "Osteoprotegerin: a novel secreted protein involved in the regulation of bone density," Cell, vol. 89, no. 2, pp. 309-319, 1997.

[30] H. Wolski, K. Drews, A. Bogacz et al., "The RANKL/RANK/ OPG signal trail: significance of genetic polymorphisms in the etiology of postmenopausal osteoporosis," Ginekologia Polska, vol. 87, no. 5, pp. 347-352, 2016.

[31] N. A. Hamdy, "Targeting the RANK/RANKL/OPG signaling pathway: a novel approach in the management of osteoporosis," Current Opinion in Investigational Drugs, vol. 8, no. 4, pp. 299-303, 2007. 
[32] L. C. Hofbauer, C. A. Kuhne, and V. Viereck, "The OPG/ RANKL/RANK system in metabolic bone diseases," Journal of Musculoskeletal \& Neuronal Interactions, vol. 4, no. 3, pp. 268-275, 2004.

[33] M. N. Weitzmann, "The role of inflammatory cytokines, the RANKL/OPG axis, and the immunoskeletal interface in physiological bone turnover and osteoporosis," Scientifica, vol. 2013, Article ID 125705, 29 pages, 2013.

[34] S. Khosla, "Minireview: the OPG/RANKL/RANK system," Endocrinology, vol. 142, no. 12, pp. 5050-5055, 2001.

[35] F. Xu, Y. Dong, X. Huang et al., "Pioglitazone affects the OPG/RANKL/RANK system and increase osteoclastogenesis," Molecular Medicine Reports, vol. 14, no. 3, pp. 22892296, 2016.

[36] K. Okamoto and H. Takayanagi, "Regulation of bone by the adaptive immune system in arthritis," Arthritis Research \& Therapy, vol. 13, no. 3, p. 219, 2011.

[37] J. Y. Li, H. Tawfeek, B. Bedi et al., "Ovariectomy disregulates osteoblast and osteoclast formation through the T-cell receptor CD40 ligand," Proceedings of the National Academy of Sciences of the United States of America, vol. 108, no. 2, pp. 768-773, 2011.

[38] K. Sato, A. Suematsu, K. Okamoto et al., "Th17 functions as an osteoclastogenic helper $\mathrm{T}$ cell subset that links T cell activation and bone destruction," The Journal of Experimental Medicine, vol. 203, no. 12, pp. 2673-2682, 2006.

[39] M. M. Zaiss, R. Axmann, J. Zwerina et al., "Treg cells suppress osteoclast formation: a new link between the immune system and bone," Arthritis \& Rheumatism, vol. 56, no. 12, pp. 4104-4112, 2007.

[40] M. M. Zaiss, B. Frey, A. Hess et al., "Regulatory T cells protect from local and systemic bone destruction in arthritis," The Journal of Immunology, vol. 184, no. 12, pp. 7238-7246, 2010.

[41] S. Y. Lee, Y. O. Jung, J. G. Ryu et al., "Intravenous immunoglobulin attenuates experimental autoimmune arthritis by inducing reciprocal regulation of Th17 and Treg cells in an interleukin-10-dependent manner," Arthritis \& Rheumatology, vol. 66, no. 7, pp. 1768-1778, 2014.

[42] H. Xu, H. Zhao, C. Lu et al., "Triptolide inhibits osteoclast differentiation and bone resorption in vitro via enhancing the production of IL- 10 and TGF- $\beta 1$ by regulatory T cells," Mediators of Inflammation, vol. 2016, Article ID 8048170, 10 pages, 2016.

[43] K. Wing, Y. Onishi, P. Prieto-Martin et al., "CTLA-4 control over Foxp $3^{+}$regulatory T cell function," Science, vol. 322, no. 5899 , pp. 271-275, 2008.

[44] R. Axmann, S. Herman, M. Zaiss et al., "CTLA-4 directly inhibits osteoclast formation," Annals of the Rheumatic Diseases, vol. 67, no. 11, pp. 1603-1609, 2008.

[45] J. Pene, S. Chevalier, L. Preisser et al., "Chronically inflamed human tissues are infiltrated by highly differentiated Th17 lymphocytes," The Journal of Immunology, vol. 180, no. 11, pp. 7423-7430, 2008.

[46] H. Takayanagi, "New immune connections in osteoclast formation," Annals of the New York Academy of Sciences, vol. 1192, no. 1, pp. 117-123, 2010.

[47] A. M. Tyagi, K. Srivastava, M. N. Mansoori, R. Trivedi, N. Chattopadhyay, and D. Singh, "Estrogen deficiency induces the differentiation of IL-17 secreting Th17 cells: a new candidate in the pathogenesis of osteoporosis," PLoS One, vol. 7, no. 9, article e44552, 2012.
[48] E. Romas, M. T. Gillespie, and T. J. Martin, "Involvement of receptor activator of $\mathrm{NF} \kappa \mathrm{B}$ ligand and tumor necrosis factor- $\alpha$ in bone destruction in rheumatoid arthritis," Bone, vol. 30, no. 2, pp. 340-346, 2002.

[49] N. Takegahara, H. Kim, H. Mizuno et al., "Involvement of receptor activator of nuclear factor- $\kappa \mathrm{B}$ ligand (RANKL)induced incomplete cytokinesis in the polyploidization of osteoclasts," The Journal of Biological Chemistry, vol. 291, no. 7, pp. 3439-3454, 2016.

[50] Y. Li, G. Toraldo, A. Li et al., "B cells and T cells are critical for the preservation of bone homeostasis and attainment of peak bone mass in vivo," Blood, vol. 109, no. 9, pp. 3839-3848, 2007.

[51] T. J. Yun, P. M. Chaudhary, G. L. Shu et al., "OPG/FDCR-1, a TNF receptor family member, is expressed in lymphoid cells and is up-regulated by ligating CD40," Journal of Immunology, vol. 161, no. 11, pp. 6113-6121, 1998.

[52] K. Titanji, A. Vunnava, A. N. Sheth et al., "Dysregulated B cell expression of RANKL and OPG correlates with loss of bone mineral density in HIV infection," PLoS Pathogens, vol. 10, no. 11, article e1004497, 2014.

[53] Y. Y. Kong, H. Yoshida, I. Sarosi et al., "OPGL is a key regulator of osteoclastogenesis, lymphocyte development and lymph-node organogenesis," Nature, vol. 397, no. 6717, pp. 315-323, 1999.

[54] G. R. Ehrhardt, A. Hijikata, H. Kitamura, O. Ohara, J. Y. Wang, and M. D. Cooper, "Discriminating gene expression profiles of memory B cell subpopulations," The Journal of Experimental Medicine, vol. 205, no. 8, pp. 1807-1817, 2008.

[55] K. Amara, E. Clay, L. Yeo et al., "B cells expressing the IgA receptor FcRL4 participate in the autoimmune response in patients with rheumatoid arthritis," Journal of Autoimmunity, vol. 81, pp. 34-43, 2017.

[56] A. I. Pesce Viglietti, P. C. Arriola Benitez, G. H. Giambartolomei, and M. V. Delpino, "Brucella abortus-infected B cells induce osteoclastogenesis," Microbes and Infection, vol. 18, no. 9, pp. 529-535, 2016.

[57] C. R. Jarry, E. F. Martinez, D. C. Peruzzo et al., "Expression of SOFAT by T- and B-lineage cells may contribute to bone loss," Molecular Medicine Reports, vol. 13, no. 5, pp. 42524258, 2016.

[58] S. Xu, Y. Zhang, B. Liu et al., "Activation of mTORC1 in B lymphocytes promotes osteoclast formation via regulation of $\beta$-catenin and RANKL/OPG," Journal of Bone and Mineral Research, vol. 31, no. 7, pp. 1320-1333, 2016.

[59] F. Azizieh, R. Raghupathy, D. Shehab, K. Al-Jarallah, and R. Gupta, "Cytokine profiles in osteoporosis suggest a proresorptive bias," Menopause, vol. 24, no. 9, pp. 1057-1064, 2017.

[60] G. Eghbali-Fatourechi, S. Khosla, A. Sanyal, W. J. Boyle, D. L. Lacey, and B. L. Riggs, "Role of RANK ligand in mediating increased bone resorption in early postmenopausal women," The Journal of Clinical Investigation, vol. 111, no. 8, pp. 1221-1230, 2003.

[61] L. C. Hofbauer, S. Khosla, C. R. Dunstan, D. L. Lacey, W. J. Boyle, and B. L. Riggs, "The roles of osteoprotegerin and osteoprotegerin ligand in the paracrine regulation of bone resorption," Journal of Bone and Mineral Research, vol. 15 , no. 1, pp. 2-12, 2000.

[62] S. Wei, M. W. Wang, S. L. Teitelbaum, and F. P. Ross, "Interleukin-4 reversibly inhibits osteoclastogenesis via inhibition 
of NF- $\kappa \mathrm{B}$ and mitogen-activated protein kinase signaling," The Journal of Biological Chemistry, vol. 277, no. 8, pp. 6622-6630, 2002.

[63] J. M. Owens, A. C. Gallagher, and T. J. Chambers, "IL-10 modulates formation of osteoclasts in murine hemopoietic cultures," Journal of Immunology, vol. 157, no. 2, pp. 936940, 1996.

[64] Y. Morita, H. Kitaura, M. Yoshimatsu et al., "IL-18 inhibits TNF- $\alpha$-induced osteoclastogenesis possibly via a T cellindependent mechanism in synergy with IL-12 in vivo," Calcified Tissue International, vol. 86, no. 3, pp. 242-248, 2010.

[65] N. J. Horwood, J. Elliott, T. J. Martin, and M. T. Gillespie, "IL12 alone and in synergy with IL-18 inhibits osteoclast formation in vitro," The Journal of Immunology, vol. 166, no. 8, pp. 4915-4921, 2001.

[66] S. Y. Kim, S. Schneeweiss, J. Liu et al., "Risk of osteoporotic fracture in a large population-based cohort of patients with rheumatoid arthritis," Arthritis Research \& Therapy, vol. 12, no. 4, p. R154, 2010.

[67] B. Mermerci Başkan, Y. Pekin Doğan, F. Sivas, H. Bodur, and K. Ozoran, "The relation between osteoporosis and vitamin $\mathrm{D}$ levels and disease activity in ankylosing spondylitis," Rheumatology International, vol. 30, no. 3, pp. 375-381, 2010.

[68] N. Davey-Ranasinghe and A. Deodhar, "Osteoporosis and vertebral fractures in ankylosing spondylitis," Current Opinion in Rheumatology, vol. 25, no. 4, pp. 509-516, 2013.

[69] S. M. Jung, K. W. Kim, C. W. Yang, S. H. Park, and J. H. Ju, "Cytokine-mediated bone destruction in rheumatoid arthritis," Journal of Immunology Research, vol. 2014, Article ID 263625, 15 pages, 2014.

[70] P. Rufus, N. Mohamed, and A. N. Shuid, "Beneficial effects of traditional Chinese medicine on the treatment of osteoporosis on ovariectomised rat models," Current Drug Targets, vol. 14, no. 14, pp. 1689-1693, 2013.

[71] H. Yamasaki, "Pharmacology of sinomenine, an antirheumatic alkaloid from Sinomenium acutum," Acta Medica Okayama, vol. 30, no. 1, pp. 1-20, 1976.

[72] Q. Wang and X. K. Li, "Immunosuppressive and antiinflammatory activities of sinomenine," International Immunopharmacology, vol. 11, no. 3, pp. 373-376, 2011.

[73] L. Liu, K. Resch, and V. Kaever, "Inhibition of lymphocyte proliferation by the anti-arthritic drug sinomenine," International Journal of Immunopharmacology, vol. 16, no. 8, pp. 685-691, 1994.

[74] Y. Cheng, J. Zhang, W. Hou et al., "Immunoregulatory effects of sinomenine on the T-bet/GATA-3 ratio and Th1/Th2 cytokine balance in the treatment of mesangial proliferative nephritis," International Immunopharmacology, vol. 9, no. 7-8, pp. 894-899, 2009.

[75] L. C. Yan, E. G. Bi, Y. T. Lou et al., "Novel sinomenine derivative 1032 improves immune suppression in experimental autoimmune encephalomyelitis," Biochemical and Biophysical Research Communications, vol. 391, no. 1, pp. 1093-1098, 2010.

[76] L. Liu, E. Buchner, D. Beitze et al., "Amelioration of rat experimental arthritides by treatment with the alkaloid sinomenine," International Journal of Immunopharmacology, vol. 18, no. 10, pp. 529-543, 1996.

[77] H. Feng, K. Yamaki, H. Takano, K. Inoue, R. Yanagisawa, and S. Yoshino, "Effect of sinomenine on collagen-induced arthritis in mice," Autoimmunity, vol. 40, no. 7, pp. 532-539, 2009.
[78] H. Zhou, Y. F. Wong, J. Wang, X. Cai, and L. Liu, "Sinomenine ameliorates arthritis via MMPs, TIMPs, and cytokines in rats," Biochemical and Biophysical Research Communications, vol. 376, no. 2, pp. 352-357, 2008.

[79] X. Li, L. He, Y. Hu et al., "Sinomenine suppresses osteoclast formation and Mycobacterium tuberculosis H37Ra-induced bone loss by modulating RANKL signaling pathways," PLoS One, vol. 8, no. 9, article e74274, 2013.

[80] B. Zhou, X. Lu, Z. Tang et al., "Influence of sinomenine upon mesenchymal stem cells in osteoclastogenesis," Biomedicine \& Pharmacotherapy, vol. 90, pp. 835-841, 2017.

[81] L. He, H. Duan, X. Li et al., "Sinomenine down-regulates TLR4/TRAF6 expression and attenuates lipopolysaccharideinduced osteoclastogenesis and osteolysis," European Journal of Pharmacology, vol. 779, pp. 66-79, 2016.

[82] K. Kawaguchi, H. Maruyama, R. Hasunuma, and Y. Kumazawa, "Suppression of inflammatory responses after onset of collagen-induced arthritis in mice by oral administration of the Citrus flavanone naringin," Immunopharmacology and Immunotoxicology, vol. 33, no. 4, pp. 723-729, 2011.

[83] E. S. Ang, X. Yang, H. Chen, Q. Liu, M. H. Zheng, and J. Xu, "Naringin abrogates osteoclastogenesis and bone resorption via the inhibition of RANKL-induced NF- $\kappa$ B and ERK activation," FEBS Letters, vol. 585, no. 17, pp. 2755-2762, 2011.

[84] J. B. Wu, Y. C. Fong, H. Y. Tsai, Y. F. Chen, M. Tsuzuki, and C. H. Tang, "Naringin-induced bone morphogenetic protein2 expression via PI3K, Akt, c-Fos/c-Jun and AP-1 pathway in osteoblasts," European Journal of Pharmacology, vol. 588, no. 2-3, pp. 333-341, 2008.

[85] K. C. Wong, W. Y. Pang, X. L. Wang et al., "Drynaria fortunei-derived total flavonoid fraction and isolated compounds exert oestrogen-like protective effects in bone," The British Journal of Nutrition, vol. 110, no. 3, pp. 475-485, 2013.

[86] W. Y. Pang, X. L. Wang, S. K. Mok et al., "Naringin improves bone properties in ovariectomized mice and exerts oestrogenlike activities in rat osteoblast-like (UMR-106) cells," British Journal of Pharmacology, vol. 159, no. 8, pp. 1693-1703, 2010.

[87] F. X. Lin, S. X. Du, D. Z. Liu et al., "Naringin promotes osteogenic differentiation of bone marrow stromal cells by upregulating Foxc2 expression via the IHH signaling pathway," American Journal of Translational Research, vol. 8, no. 11, pp. 5098-5107, 2016.

[88] J. H. Xu, M. Yao, J. Ye et al., "Bone mass improved effect of icariin for postmenopausal osteoporosis in ovariectomyinduced rats: a meta-analysis and systematic review," Menopause, vol. 23, no. 10, pp. 1152-1157, 2016.

[89] L. Xue, Y. Jiang, T. Han et al., "Comparative proteomic and metabolomic analysis reveal the antiosteoporotic molecular mechanism of icariin from Epimedium brevicornu maxim," Journal of Ethnopharmacology, vol. 192, pp. 370-381, 2016.

[90] G. Chen, C. Wang, J. Wang et al., "Antiosteoporotic effect of icariin in ovariectomized rats is mediated via the $\mathrm{Wnt} / \beta$ catenin pathway," Experimental and Therapeutic Medicine, vol. 12, no. 1, pp. 279-287, 2016.

[91] S. Qin, W. Zhou, S. Liu, P. Chen, and H. Wu, "Icariin stimulates the proliferation of rat bone mesenchymal stem cells via ERK and p38 MAPK signaling," International Journal of Clinical and Experimental Medicine, vol. 8, no. 5, pp. 71257133, 2015. 
[92] G. W. Li, Z. Xu, S. X. Chang, H. Nian, X. Y. Wang, and L. D. Qin, "Icariin prevents ovariectomy-induced bone loss and lowers marrow adipogenesis," Menopause, vol. 21, no. 9, pp. 1007-1016, 2014.

[93] H. Liu, Y. Xiong, X. Zhu et al., "Icariin improves osteoporosis, inhibits the expression of PPAR $\gamma, \mathrm{C} / \mathrm{EBP} \alpha, \mathrm{FABP} 4 \mathrm{mRNA}$, N1ICD and jagged1 proteins, and increases Notch 2 mRNA in ovariectomized rats," Experimental and Therapeutic Medicine, vol. 13, no. 4, pp. 1360-1368, 2017.

[94] H. Cao, Y. Zhang, W. Qian et al., "Effect of icariin on fracture healing in an ovariectomized rat model of osteoporosis," Experimental and Therapeutic Medicine, vol. 13, no. 5, pp. 2399-2404, 2017.

[95] Y. Wu, L. Cao, L. Xia et al., "Evaluation of osteogenesis and angiogenesis of icariin in local controlled release and systemic delivery for calvarial defect in ovariectomized rats," Scientific Reports, vol. 7, no. 1, article 5077, 2017.

[96] T. P. Hsieh, S. Y. Sheu, J. S. Sun, M. H. Chen, and M. H. Liu, "Icariin isolated from Epimedium pubescens regulates osteoblasts anabolism through BMP-2, SMAD4, and Cbfa1 expression," Phytomedicine, vol. 17, no. 6, pp. 414-423, 2010.

[97] W. Liang, M. Lin, X. Li et al., "Icariin promotes bone formation via the BMP-2/Smad4 signal transduction pathway in the hFOB 1.19 human osteoblastic cell line," International Journal of Molecular Medicine, vol. 30, no. 4, pp. 889-895, 2012.

[98] E. M. Tan, L. Li, I. R. Indran, N. Chew, and E. L. Yong, "TRAF6 mediates suppression of osteoclastogenesis and prevention of ovariectomy-induced bone loss by a novel prenylflavonoid," Journal of Bone and Mineral Research, vol. 32, no. 4, pp. 846-860, 2017.

[99] T. Wu, T. Shu, L. Kang et al., "Icaritin, a novel plant-derived osteoinductive agent, enhances the osteogenic differentiation of human bone marrow- and human adipose tissue-derived mesenchymal stem cells," International Journal of Molecular Medicine, vol. 39, no. 4, pp. 984-992, 2017.

[100] H. Sheng, X. F. Rui, C. J. Sheng et al., "A novel semisynthetic molecule icaritin stimulates osteogenic differentiation and inhibits adipogenesis of mesenchymal stem cells," International Journal of Medical Sciences, vol. 10, no. 6, pp. 782-789, 2013.

[101] H. Ha, J. Ho, S. Shin et al., "Effects of Eucommiae Cortex on osteoblast-like cell proliferation and osteoclast inhibition," Archives of Pharmacal Research, vol. 26, no. 11, pp. 929-936, 2003.

[102] R. Zhang, Z. G. Liu, C. Li et al., "Du-Zhong (Eucommia ulmoides Oliv.) cortex extract prevent OVX-induced osteoporosis in rats," Bone, vol. 45, no. 3, pp. 553-559, 2009.

[103] Y. Li, M. J. Wang, S. Li et al., "Effect of total glycosides from Eucommia ulmoides seed on bone microarchitecture in rats," Phytotherapy Research, vol. 25, no. 12, pp. 1895-1897, 2011.

[104] W. Zhang, T. Fujikawa, K. Mizuno et al., "Eucommia leaf extract (ELE) prevents OVX-induced osteoporosis and obesity in rats," The American Journal of Chinese Medicine, vol. 40, no. 04, pp. 735-752, 2012.

[105] X. L. Tan, Y. H. Zhang, J. P. Cai, L. H. Zhu, W. J. Ge, and X. Zhang, "5-(Hydroxymethyl)-2-furaldehyde inhibits adipogenic and enhances osteogenic differentiation of rat bone mesenchymal stem cells," Natural Product Communications, vol. 9, no. 4, pp. 529-532, 2014.
[106] R. Zhang, Y. L. Pan, S. J. Hu, X. H. Kong, W. Juan, and Q. B. Mei, "Effects of total lignans from Eucommia ulmoides barks prevent bone loss in vivo and in vitro," Journal of Ethnopharmacology, vol. 155, no. 1, pp. 104-112, 2014.

[107] J. Y. Wang, Y. Yuan, X. J. Chen et al., "Extract from Eucommia ulmoides Oliv. ameliorates arthritis via regulation of inflammation, synoviocyte proliferation and osteoclastogenesis in vitro and in vivo," Journal of Ethnopharmacology, vol. 194, pp. 609-616, 2016.

[108] G. P. Xie, N. Jiang, S. N. Wang et al., “Eucommia ulmoides Oliv. bark aqueous extract inhibits osteoarthritis in a rat model of osteoarthritis," Journal of Ethnopharmacology, vol. 162, pp. 148-154, 2015.

[109] S. H. Kwon, S. X. Ma, J. Y. Hwang et al., "The antiinflammatory activity of Eucommia ulmoides Oliv. bark. involves NF- $\kappa \mathrm{B}$ suppression and Nrf2-dependent $\mathrm{HO}-1$ induction in BV-2 microglial cells," Biomolecules \& Therapeutics, vol. 24, no. 3, pp. 268-282, 2016.

[110] X. Dai, Q. Huang, B. Zhou, Z. Gong, Z. Liu, and S. Shi, "Preparative isolation and purification of seven main antioxidants from Eucommia ulmoides Oliv. (Du-zhong) leaves using HSCCC guided by DPPH-HPLC experiment," Food Chemistry, vol. 139, no. 1-4, pp. 563-570, 2013.

[111] M. M. Bai, W. Shi, J. M. Tian et al., "Soluble epoxide hydrolase inhibitory and anti-inflammatory components from the leaves of Eucommia ulmoides Oliver (Duzhong)," Journal of Agricultural and Food Chemistry, vol. 63, no. 8, pp. 2198-2205, 2015.

[112] T. Oliveira, C. A. Figueiredo, C. Brito et al., "Allium cepa L. and quercetin inhibit RANKL/porphyromonas gingivalis LPS-induced osteoclastogenesis by downregulating NF- $\kappa \mathrm{B}$ signaling pathway," Evidence-Based Complementary and Alternative Medicine, vol. 2015, Article ID 704781, 11 pages, 2015.

[113] K. S. Shim, H. Ha, T. Kim, C. J. Lee, and J. Y. Ma, “Orostachys japonicus suppresses osteoclast differentiation by inhibiting NFATc1 expression," The American Journal of Chinese Medicine, vol. 43, no. 05, pp. 1013-1030, 2015.

[114] A. Wattel, S. Kamel, C. Prouillet et al., "Flavonoid quercetin decreases osteoclastic differentiation induced by RANKL via a mechanism involving NF $\kappa \mathrm{B}$ and AP-1," Journal of Cellular Biochemistry, vol. 92, no. 2, pp. 285-295, 2004.

[115] M. Tsuji, H. Yamamoto, T. Sato et al., "Dietary quercetin inhibits bone loss without effect on the uterus in ovariectomized mice," Journal of Bone and Mineral Metabolism, vol. 27, no. 6, pp. 673-681, 2009.

[116] J. T. Woo, H. Nakagawa, M. Notoya et al., "Quercetin suppresses bone resorption by inhibiting the differentiation and activation of osteoclasts," Biological and Pharmaceutical Bulletin, vol. 27, no. 4, pp. 504-509, 2004.

[117] M. Yamaguchi and M. Weitzmann, "Quercetin, a potent suppressor of NF- $\kappa \mathrm{B}$ and Smad activation in osteoblasts," International Journal of Molecular Medicine, vol. 28, no. 4, pp. 521-525, 2011.

[118] C. Guo, G. Q. Hou, X. D. Li et al., "Quercetin triggers apoptosis of lipopolysaccharide (LPS)-induced osteoclasts and inhibits bone resorption in RAW264.7 cells," Cellular Physiology and Biochemistry, vol. 30, no. 1, pp. 123-136, 2012.

[119] M. Yamaguchi, R. Hamamoto, S. Uchiyama, and K. Ishiyama, "Effects of flavonoid on calcium content in femoral tissue culture and parathyroid hormone-stimulated 
osteoclastogenesis in bone marrow culture in vitro," Molecular and Cellular Biochemistry, vol. 303, no. 1-2, pp. 83-88, 2007.

[120] X. C. Wang, N. J. Zhao, C. Guo, J. T. Chen, J. L. Song, and L. Gao, "Quercetin reversed lipopolysaccharide-induced inhibition of osteoblast differentiation through the mitogenactivated protein kinase pathway in MC3T3-E1 cells," Molecular Medicine Reports, vol. 10, no. 6, pp. 3320-3326, 2014.

[121] F. Li, X. Yang, Y. Yang et al., "Antiosteoporotic activity of echinacoside in ovariectomized rats," Phytomedicine, vol. 20, no. 6, pp. 549-557, 2013.

[122] Y. B. Niu, Y. H. Li, X. H. Kong et al., “The beneficial effect of Radix Dipsaci total saponins on bone metabolism in vitro and in vivo and the possible mechanisms of action," Osteoporosis International, vol. 23, no. 11, pp. 2649-2660, 2012.

[123] R. Zhang, S. J. Hu, C. Li, F. Zhang, H. Q. Gan, and Q. B. Mei, "Achyranthes bidentata root extract prevent OVX-induced osteoporosis in rats," Journal of Ethnopharmacology, vol. 139, no. 1, pp. 12-18, 2012.

[124] Y. Shen, Y. Q. Li, S. P. Li, L. Ma, L. J. Ding, and H. Ji, “Alleviation of ovariectomy-induced osteoporosis in rats by Panax notoginseng saponins," Journal of Natural Medicines, vol. 64, no. 3, pp. 336-345, 2010.

[125] S. Chawalitpong, N. Sornkaew, A. Suksamrarn, and T. Palaga, "Diarylheptanoid from Curcuma comosa Roxb. suppresses RANKL-induced osteoclast differentiation by decreasing NFATc1 and c-Fos expression via MAPK pathway," European Journal of Pharmacology, vol. 788, pp. 351-359, 2016.

[126] X. Peng, J. He, J. Zhao et al., "Polygonatum sibiricum polysaccharide promotes osteoblastic differentiation through the ERK/GSK-3 $\beta / \beta$-catenin signaling pathway in vitro," Rejuvenation Research, 2017.

[127] W. Zhou, X. Cheng, and Y. Zhang, "Effect of Liuwei Dihuang decoction, a traditional Chinese medicinal prescription, on the neuroendocrine immunomodulation network," Pharmacology \& Therapeutics, vol. 162, pp. 170-178, 2016.

[128] F. Jian, Z. Yongxiang, and R. Xiangbin, "Effect of Liuwei Dihuang decoction, a traditional Chinese medicinal tonic prescription, on the immune function of adjuvant arthritis rats," Pharmacology and Clinics of Chinese Materia Medica, vol. 1, pp. 3-5, 2001.

[129] F. Jian, Z. Yongxiang, and R. Xiangbin, "Effect of Liuwei Dihuang decoction on the cytokine expression in splenocytes in AA rats," China Journal of Chinese Materia Medica, vol. 2, pp. 56-59, 2001.

[130] J. R. Ge, L. H. Xie, J. Chen et al., "Liuwei Dihuang pill (六味地 黄丸) treats postmenopausal osteoporosis with Shen (kidney) yin deficiency via Janus kinase/signal transducer and activator of transcription signal pathway by up-regulating cardiotrophin-like cytokine factor 1 expression," Chinese Journal of Integrative Medicine, 2016.

[131] B. Xia, B. Xu, Y. Sun et al., "The effects of Liuwei Dihuang on canonical Wnt $/ \beta$-catenin signaling pathway in osteoporosis," Journal of Ethnopharmacology, vol. 153, no. 1, pp. 133-141, 2014.
[132] M. J. Liu, L. I. Yan, J. H. Pan et al., "Effects of zuogui pill (左归丸) on Wnt singal transduction in rats with glucocorticoid-induced osteoporosis," Journal of Traditional Chinese Medicine, vol. 31, no. 2, pp. 98-102, 2011.

[133] N. Lai, Z. Zhang, B. Wang et al., "Regulatory effect of traditional Chinese medicinal formula Zuo-Gui-Wan on the Th17/Treg paradigm in mice with bone loss induced by estrogen deficiency," Journal of Ethnopharmacology, vol. 166, pp. 228-239, 2015. 


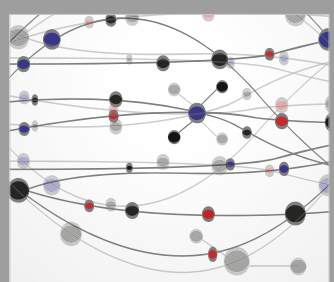

The Scientific World Journal
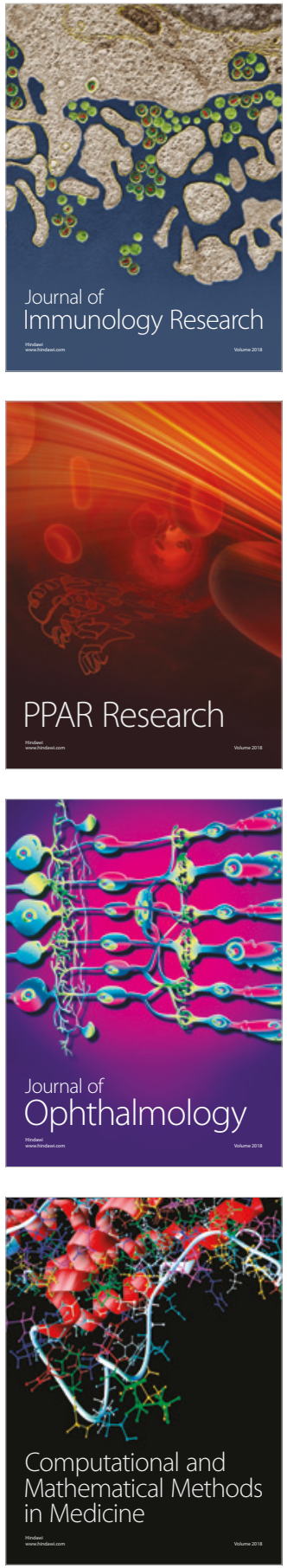

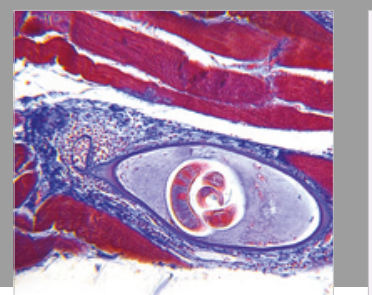

Gastroenterology Research and Practice

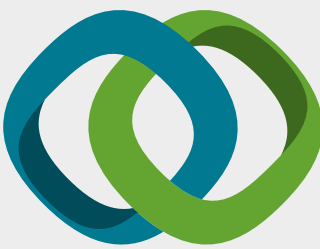

\section{Hindawi}

Submit your manuscripts at

www.hindawi.com
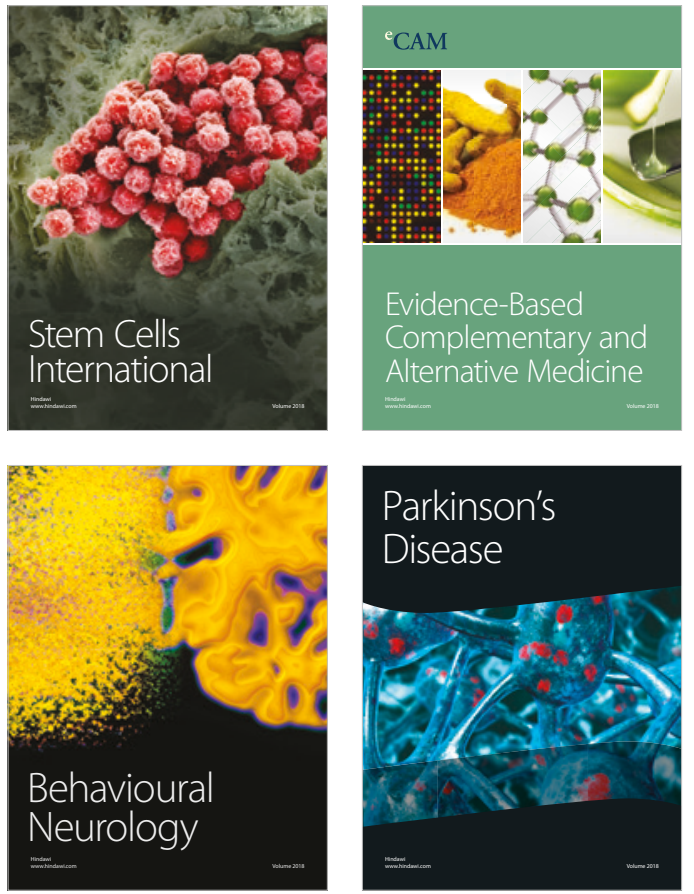

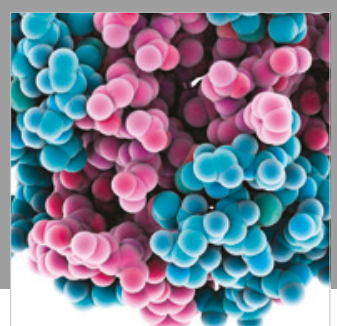

ournal of

Diabetes Research

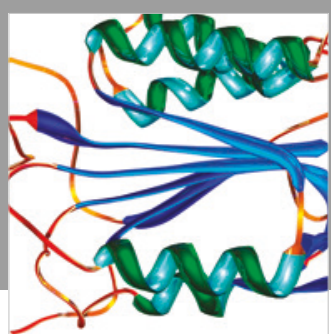

Disease Markers
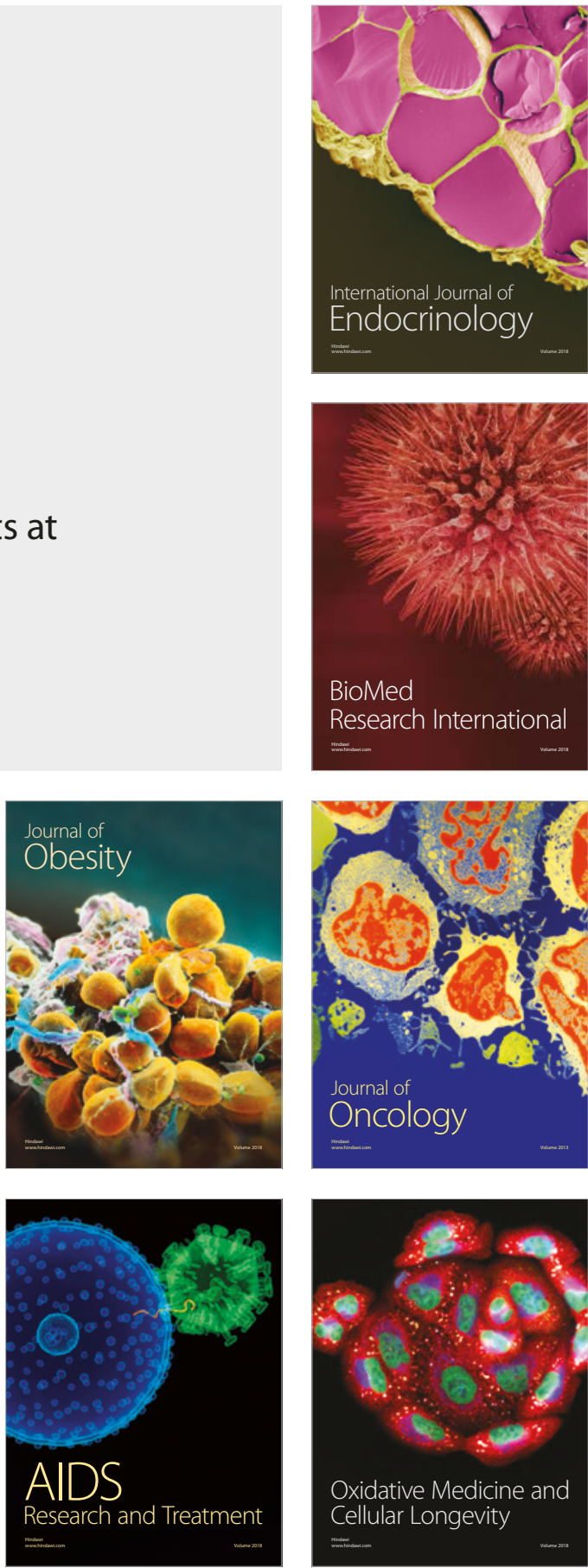